\title{
The Price of Services
}

\author{
Justin O'Sullivan, David Edmond, and Arthur H.M. ter Hofstede \\ Business Process Management Program, \\ Faculty for Information Technology, \\ Queensland University of Technology, \\ GPO Box 2434, \\ Brisbane QLD 4001, Australia \\ justin@service-description.com
}

\begin{abstract}
If we accept that service providers and service users all operate with autonomy in some form of market place, then a necessary prerequisite for service discovery and engagement is the description of the non-functional properties of a service. Price acts as one of the key nonfunctional properties used in choosing candidate services. Conventional services describe prices using several approaches (e.g. fixed price, price ranges, proportional pricing, dynamic price mechanisms). Furthermore, there are associated concepts such as price matching, price granularity, taxes and reward schemes that might need to be taken into consideration. This paper offers a discussion of the non-functional property of price. By incorporating some information about price, service descriptions will move away from the narrow distributed computing view of web services, enabling greater reasoning with respect to service descriptions.
\end{abstract}

\section{Introduction}

Through media such as newspapers, letterbox flyers, corporate brochures and television we are regularly confronted with descriptions for conventional services. These representations vary in the terminology utilised, the depth of the description, and the aspects of the service that are characterised. Existing service catalogues provide little relief for service requestors from the burdensome task of discovering, comparing and substituting services. Add to this environment the rapidly evolving area of web services with its associated surfeit of standards, and the result is a considerably fragmented approach to the description of services. It leaves the vision of the Semantic Web [1] somewhat clouded.

We have previously claimed that non-functional properties are an essential component of the characterisation of any service [2]. In [3] we present a discussion of many non-functional properties that can be used to improve discovery, comparison and service substitution. The non-functional properties we capture include availability (both temporal and locative), payment, price, discounts, obligations, rights, penalties, trust, security, and quality. This content has been published on the Web as a set of navigable models (http://www . service-description.com/). To develop these models we undertook a significant analysis of services from numerous domains. We have extracted hundreds 
of non-functional related properties that have been subjected to criteria before inclusion in our models. This work is an attempt to narrow the void between the functionally focused web service description standards and the non-functional description of services. It is our opinion that the semantic richness of the nonfunctional properties of services is not being exploited. We refer to this as "semantic myopia" 4].

The rest of the paper is structured as follows. In section 2 we provide an insight into our motivations whilst also positioning our work with respect to other research. Next, in section 3 , we present a discussion of the non-functional property of price. Finally, we present our conclusions in section 4. We are unable to portray our entire formal taxonomy within the space considerations of this paper. See http://www.service-description.com/ for the complete taxonomy.

\section{Motivations}

Our primary motivation is to provide a necessary pre-requisite to automated service discovery, service selection and service substitution. We propose some sample questions that we put to existing web service description standards as a means of highlighting our concerns. What percentage deduction does the service provider offer when they are willing to match the price of an equivalent service from another service provider? How many reward scheme points are acquired when paying a particular price for a service? These are terms that service requestors (i.e. people, organisations) currently utilise when discovering services. Are web services so different from conventional services? Removing the "tunnel vision" of service descriptions to include both web and conventional services results in the ability to compare both types of services. We prefer not to distinguish between conventional services and web services. We are motivated to ensure that the criteria used to evaluate conventional services are also available for web services.

To achieve these benefits, a service description technique is required that is capable of expressing the functional and non-functional aspects of services. We subscribe to the notion that non-functional properties are constraints over the functionality [5]. Existing semantic web services initiatives, whilst offering the ability to capture the non-functional properties, have lacked the depth of description that we advocate. The OWL web service ontology (OWL-S) 6] offers placeholders for the description of non-functional service properties, along with a minimal number of specific non-functional properties. The Web Services Modelling Ontology (WSMO) 7] uses Dublin Core metadata and a version number as the core properties, then extends these to include web service specific categories of non-functional properties (e.g. performance, security, financial). Our approach to the description of non-functional properties is complementary to both OWL-S and WSMO. We now offer our discussion of the non-functional property of price. 


\section{Price}

We interchangeably refer to price as cost: cost being mostly the view from a service requestor perspective, whilst price is the view from the service provider perspective. Within this paper we refer to price as the amount being charged for a service. We believe that the pricing of a service is an obligation of the service provider, one of many obligations involved with service request and provision. We refer to it as a pricing obligation since there are costs involved in supplying the service to the requestor and therefore the provider would normally attempt to recoup these costs (plus a margin).

Examples of price descriptions include:

- Carpet cleaning: a carpet dry cleaning service offers 3 rooms cleaned for $\$ 89$ AUD (where the maximum room size is $13 \mathrm{sq} \mathrm{m}$, and subject to inspection of the carpet condition). They also offer 2 rooms for $\$ 69$ AUD with additional rooms $\$ 25$ AUD per room. Four rooms cost $\$ 110$ AUD.

- Newspaper delivery: a newsagent offers home delivery of newspapers daily for $\$ 7.20$ AUD per week (i.e. 7 days for $\$ 7.20$ AUD).

- Accommodation: a hotel in Surfers Paradise is offering a room for $\$ 82.50$ AUD per adult twin share.

From these examples we can see that prices are complex entities. They are not always easily captured as a simple dollar value in a certain currency. Prices become quite domain specific when granularities (e.g. per room) are applied. Certain complex conditions may also surround the eligibility of a service requestor to receive the advertised price. To this end we consider that a pricing obligation can be considered to wrap the price of a service with many other important non-functional properties. These may include:

- Price validity - this provides a where and when scoping of the price's availability. Using our temporal models (defined in [3]) the temporal validity can be specified as an anchored or recurring interval, an instant or a date. We also capture the location as the pricing obligation may be specific to a limited number of the locations where a service can be requested from. For example, an online retailer may offer a priority shipping service as an alternative to standard shipping. For each region within a particular country, the retailer would be able to state the price that is available for a particular timeframe.

- Conditions - these relate to any specific requirements or restrictions to the price, or to the refund for the price paid for a service. Conditions are complex entities that require a concrete representation. We prefer to identify conditions through URL referrals. Refund conditions are common for transportation services such as plane tickets where they state that a ticket may not be refundable, or may only be refunded within a particular timeframe.

- Refund procedure - associated with the specifying of refund conditions it may also be necessary for a service provider to state a refund procedure. This procedure is used by service requestors to enact the refund process. We consider procedures to be a sequence of steps that are followed to achieve an outcome. 
- Negotiability - sometimes the service provider may advertise a price but be willing to accept a lesser amount. Our model allows the provider to state that they are willing to negotiate on price.

- Price customisation - this allows the provider to explain that its service is highly customisable, and therefore the actual price cannot be expressed (e.g. a landscaping service may not be able to express the price until they have an understanding of the requestor's block of land and their objectives). This does not reduce the usefulness of the service description as the service provider is still capable of expressing the other pricing obligation related properties within this list.

- Relationship obligation - this allows the service provider to state that a relationship is required before they will commit to a price and its surrounding non-functional properties (e.g. conditions, discounts). It is possible within our model to specify an obligation that refers to the need to have a relationship with the service provider to receive the service output.

- Payee discounts - we provide an in-depth discussion of discounts in [3] but provide a link within our pricing obligation model to one specific type of discount, those related to who the payee is. This might include a person from a particular age group (e.g. the elderly), those with membership to a particular body, or even a shareholder of a company.

We consider that the pricing obligation of the service provider, in conjunction with the price, produces a new entity that we refer to as the "ServicePrice". We attach further information to this entity later in this section (e.g. tax, price granularity, price modifier). We also consider that after stating a price (e.g. 10 nights at $\$ 150$ USD per night) the service provider might attach the price for additional invocations (e.g. each extra night is $\$ 100$ USD per night). We assert that every price is one of the following kinds:

- Absolute price - this contains a specific amount and a currency. For example \$10 AUD represents ten (10) Australian dollars.

- Proportional price - this represents a percentage value with respect to a certain item. For example, the price of entering a managed fund might be $2.5 \%$ of the value being invested into the fund.

- Ranged price - Ranged prices are further subdivided into one of two types:

- Ranged absolute - a ranged absolute price contains a from and to value that are both absolute prices. For example, a service provider may prefer to provide a ranged price rather than a specific price (e.g. $\$ 150,000$ USD - $\$ 175,000$ USD).

- Ranged proportional - a ranged proportional price contains a from and to value that are both proportional prices. For example, a service provider may state the cost of its service as a range between $1.5 \%$ and $3 \%$ of the final sale price.

- Dynamic price - this form of pricing captures mechanisms like auctions, where the price is determined by a market's natural supply and demand. We capture the type of mechanism (such as English auction, Dutch auction etc), the conditions associated with using the mechanism, the location and 
temporal availability of the mechanism, and a reserve price (as either an absolute or proportional price). We provide a link to the provider of the dynamic pricing mechanism (e.g. eBay). An example of a service that could be auctioned is advertising space on web sites.

Price also includes an item granularity that is applicable to all types of prices (e.g. per person per night). The granularity of the item reflects one or more units of measure. We foresee the use of common granularities such as time (hour, minute, second, day, month, year, night, week, fortnight), weight (gram, kilogram, tonne), volume (cubic metre), area (metres squared, square metres), length (millimetre, centimetre, metre, kilometre), byte (kilobyte, megabyte, gigabyte), and person (adult, child, infant, pensioner, senior). These granularities could be extended further to support notions such as a room. This caters for services such as our carpet dry cleaning or accommodation examples.

All prices have a modifier that quantifies the price being specified. In using the term "quantify" we are referring to it in a logical sense rather than in an arithmetic sense. We have provided four example modifiers: exact, limited to (the price will not go higher than the amount specified), inclusive (intended for ranges of values) and from (the price starts at this amount and will go higher depending on how the service is configured by the requestor). Prices may include a component that is tax related. Service providers can choose to state their price as inclusive or exclusive of a tax item. If a tax item is captured, then a tax percentage is attached. For example, Australians are taxed at a rate of $10 \%$ on the majority of goods and services they purchase under the Goods and Services Tax (GST). Similar taxes include the Value Added Tax (VAT). Tax is applicable to a particular region.

Some services offer a price based on the criterion that the service requestor also requests the use of another service. An example is that the carpet cleaning service provider will offer their carpet protection service only when addition cleaning services are purchased. A service price may also provide either the service requestor or the service provider with one or more rights with respect to the service. Rights are outlined in more depth in 3]. We provide a price matching facility within our price model. Some service providers advertise that they are willing to match or better the price of another competitor. For this type of service provider we allow the attachment of a percentage which indicates what they are willing to improve competitor offers by (e.g. 5\%).

Some service providers choose to reward service requestors using loyalty schemes. We attach to the price of a service the possibility of accumulating rewards under a reward scheme. Reward schemes can be provided by the service provider or by a third-party. Our model allows a service to attach a number of reward points to the invocation of the service, based on the service price that is paid (remembering that prices have a temporal and a locative availability). Reward points are only available during certain temporal intervals, or on a particular date, as well as being surrounded by some conditions. In a complementary manner we allow the service provider to state that they accept rewards scheme points as payment for a service. 


\section{Conclusions}

Our approach seeks to offer a domain-independent method for describing the non-functional properties of both conventional and web services. Due to space limitations we have presented only part of the non-functional property of price. Our recent work 3 provides the same level of descriptive depth for other nonfunctional properties such as availability (temporal and locative), payment, obligations, rights, discounts, penalties, trust, security and quality. We feel that this approach is complementary to existing semantic web service initiatives such as WSMO and OWL-S.

The non-functional properties of services introduce complexity to the description of services but their inclusion is crucial to the automation of service discovery, comparison and substitution. We have stated in this paper our belief that two challenges confront the future of service description - overcoming web service tunnel vision and overcoming semantic myopia. That is, choosing to ignore both the rich history of conventional services, and the non-functional properties of services (perhaps through deferring to domain specific ontologies, or by a continued functional focus). Our work provides an opportunity for expressing the non-functional properties of services using a single technique for both conventional and web services, whilst also addressing our stated concerns.

\section{References}

1. Berners-Lee, T., Fielding, R.T., Masinter, L.: Uniform Resource Identifiers (URI): Generic Syntax (1998) Available from http://www.ietf.org/rfc/rfc2396.txt, accessed on 19-Jun-2001.

2. O'Sullivan, J., Edmond, D., Hofstede, A.t.: What's in a service?: Towards accurate description of non-functional service properties. Distributed and Parallel Databases Journal - Special Issue on E-Services 12 (2002) 117-133

3. O'Sullivan, J., Edmond, D., Hofstede, A.H.t.: Formal description of nonfunctional service properties. Technical FIT-TR-2005-01, Queensland University of Technology, Brisbane (2005) Available from http://www.citi.qut.edu.au/about/ research_pubs/technical/non-functional.jsp, accessed on 15-Feb-2005.

4. O'Sullivan, J., Edmond, D., Hofstede, A.H.t.: Two main challenges in service description: Web service tunnel vision and Semantic myopia. In: W3C Workshop on Frameworks for Semantics in Web Services, Innsbruck, Austria (2005)

5. Chung, L.: Non-Functional Requirements for Information System Design. In Andersen, R., Bubenkor, J.A., Sølvberg, A., eds.: Proceedings of the 3rd International Conference on Advanced Information Systems Engineering - CAiSE'91. Lecture Notes in Computer Science, Trodheim, Norway, Springer-Verlag (1991) 5-30

6. OWL-S Coalition: OWL-S Web Service Ontology (2004) Available from http://www.daml.org/services/owl-s/1.1/, accessed on 21-Nov-2004.

7. Bruijn, J.d., Bussler, C., Fensel, D., Kifer, M., Kopecky, J., Lara, R., Oren, E., Polleres, A., Stollberg, M.: Web Services Modeling Ontology (WSMO) - Working Draft 21st November 2004 (2004) Available from http://www.wsmo.org/2004/d2/ v1.1/20041121/, accessed on 22-Nov-2004. 